\title{
Dinámica de la biomasa microbiana y su relación con la respiración y el nitrógeno del suelo en tierras agrícolas en el valle del Mantaro
}

\section{Dynamics of microbial biomass and its relationships with soil respiration and total nitrogen in agricultural land in Mantaro Valley}

\author{
Amanda Hinostroza ${ }^{1}$ Julio Malca ${ }^{2}$ Luis Suarez ${ }^{3}$
}

http://dx.doi.org/10.21503/CienciayDesarrollo.2013.v16i1.02

\section{RESUMEN}

Se estudió el efecto del abonamiento orgánico con estiércol de ganado vacuno, ovino, de cuy, fertilización química y sin abonamiento sobre la variación del nitrógeno, variación de la biomasa microbiana del suelo y la respiración del suelo en cultivos de papa (Solanum tuberosum) y maíz (Zea mays) durante la campaña agrícola 2009 en cuatro localidades del valle del Mantaro: El Mantaro, Chupaca, Santa Ana y Huayao, bajo diseño experimental de bloques completamente aleatorios con arreglo factorial. La biomasa microbiana se cuantificó mediante el método de fumigación - extracción, y la respiración basal del suelo $(\mathrm{RB})$ se determinó mediante incubación. Los resultados indican tendencia polinomial cúbica en la variación del nitrógeno, mostrando una continua disminución hacia los 60 días y un ligero ascenso hacia los 90 días.

De otro lado no, existe una relación definida entre la biomasa microbiana y la respiración del suelo. Se han determinado valores de $\mathrm{RB}$ desde 0,02 a 3,26y desde 0,14 a $2,34 \mathrm{mg} \mathrm{CO}_{2} / \mathrm{ha} / \mathrm{h}$ en cultivos de papa y maíz, respectivamente; valores que indican actividad microbiológica de muy baja a media en la mayoría de suelos y tratamientos, presentándose la mayor tasa de respiración durante el mes de diciembre, periodo con mayor temperatura en el suelo con respecto a los meses de febrero y marzo, esto comprueba que a mayor temperatura mayor respiración basal.

Palabras clave: Biomasa microbiana del suelo. Respiración del suelo. Nitrógeno en el suelo. Valle del Mantaro.

\section{ABSTRACT}

It was studied the effect of organic fertilization with manure of cattle, sheep, guinea pig, composting chemical and without fertilization on nitrogen variation, variation of soil microbial biomass and soil respiration in potato crops (Solanum tuberosum) and corn (Zea mays) during the cropping season 2009 at four locations in the Mantaro Valley: Mantaro, Chupaca, Santa Ana and Huayao under experimental design of completely random block with factorial arrangement. Microbial biomass was quantified by the method of spraying - removal and basal soil respiration was determined by incubation. The results indicate cubic polynomial trend in the variation of nitrogen, showing a steady decrease to 60 days and a slight increase to 90 days.

\footnotetext{
1 Ingeniera Ambiental, Universidad Alas Peruanas - Filial Huancayo.email: nahi_beth@uap.edu.pe

2 Ingeniero Agrónomo. Docente de Estadística y Metodología de la Investigación de la E.A.P. de Ingenieria Ambiental de la Universidad Alas Peruanas - Filial Huancayo. email: jcesarmjpe@yahoo.es

3 Ingeniero químico. Ex Director de la E.A.P. de Ingenieria Ambiental de la Universidad Alas Peruanas - Filial Huancayo. email: luchinsuarez@yahoo.com
} 
On the other hand there is not a definite relationship between microbial biomass and soil respiration. Values of Basal Soil Respiration have been determined from 0.02 to 3.26 and from 0.14 to $2.34 \mathrm{mg}$ $\mathrm{CO} 2 / \mathrm{ha} / \mathrm{h}$ in potato crops and maize, respectively, values that indicate microbiological activity of very low to medium in most soils and treatments, presenting the highest rate of respiration during December, a period with higher temperature on the ground in February and March, this proves that the higher temperature increased basal respiration.

Keywords: Soil microbial biomass, soil respiration, soil nitrogen, Mantaro Valley.

\section{INTRODUCCIÓN}

El estudio de la biomasa microbiana del suelo (BMS) es de fundamental importancia para estudiar un rango diverso de procesos edáficos, incluyendo aquellos involucrados en el ciclaje de nutrientes, la descomposición de la materia orgánica, calidad de suelo y para aplicaciones de modelamiento y monitoreo (Jenkinson 1988). La BMS, conceptualmente es la parte viva de la materia orgánica del suelo y forma parte de la reserva energética del suelo. Comprende una fuente potencial de $\mathrm{C}, \mathrm{N}, \mathrm{P}$ y otros nutrientes para las plantas. La respiración del suelo: Nos sirve como indicador de la actividad microbiana y de las raíces.

La materia orgánica nos da información sobre la cantidad de carbono orgánico presente en el suelo; el nitrógeno total, nos da información sobre la cantidad de nitrógeno disponible presente en el suelo útil para la planta; la biomasa microbiana de carbono, sirve como indicador de la actividad microbiana y su relación con el elemento carbono.

La actividad microbiana del suelo puede ser estimada indirectamente con la determinación de la respiración basal. Ésta consiste en determinar la producción de $\mathrm{O}_{2}$ en el medio o bien la concentración de $\mathrm{CO}_{2}$ desprendido (función de la actividad biológica y del contenido del suelo en carbono orgánico fácilmente mineralizable), mediante la técnica de incubación estática que captura el producto de mineralización en una solución alcalina durante un periodo de tiempo bajo condiciones ambientales óptimas (Alef y Nannipieri, 1995; García et al., 2003).

Según Moreira y Siqueira (2002), la respiración es uno de los parámetros más antiguos para cuantificar la actividad microbiana. Ella representa la oxidación de la materia orgánica hasta la formación de dióxido de carbono $\left(\mathrm{CO}_{2}\right)$ por organismos aeróbicos del suelo, que por lo tanto utilizan $\mathrm{O}_{2}$ como aceptor final de electrones, hasta el $\mathrm{CO}_{2}$. La respiración del suelo es altamente variable, tanto espacialmente como estacionalmente, y está fuertemente afectada por condiciones de humedad y temperatura.

La actividad biológica es un reflejo directo de la degradación de materia orgánica del suelo. Esta degradación indica que están sucediendo dos procesos: (1) pérdida de carbono del suelo. y (2), entrega de nutrientes (Parkin et al.,1996). Un índice de respiración edáfica óptimo, que balancee los aspectos perjudiciales a largo plazo de la pérdida de carbono y la entrega de nutrientes del suelo debe ser definido (USDA, 1999).

Los factores que afectan el crecimiento también influyen en la respiración en el mismo grado. Los principales factores conocidos que afectan la respiración aeróbica de bacterias heterotróficas son: temperatura, oxígeno disuelto, humedad del suelo, pH, y nutrientes (Krebs, 2003).Un incremento de $10^{\circ} \mathrm{C}$ a partir de temperaturas sub-óptimas, 
puede duplicar la respiración y crecimiento de las bacterias. Muchas bacterias encontradas en el suelo crecen mejor entre 30 a $35^{\circ} \mathrm{C}$ (Thamdrupet et al., 1998).

La humedad, la intensidad de la radiación solar por unidad de superficie, la evaporación, convección, la exposición, la sombra y el aislamiento son importantes factores que regulan la temperatura del suelo (Hagan, R y McCalla 1952, citado por J., Ochse, et al, 1991).

En climas cálidos las tasas de respiración son mayores y varían con el $\mathrm{pH}$ del suelo, contenido de humedad, suplemento de $\mathrm{O}_{2}$ y disponibilidad de N. Además, son mayores cerca de la superficie del suelo, debido a altas concentraciones de MO y a la disponibilidad de oxígeno.Rangos usuales de respiración se encuentran entre $10-$ $100 \mathrm{kgCO}_{2} / \mathrm{ha} / \mathrm{d}$, pero la mayoría de suelos se encuentran en el rango $25-40 \mathrm{kgCO}_{2} / \mathrm{ha} / \mathrm{d}$. Bowman y Delfino (1980) encontraron en laboratorio, en sistemas donde adecuadas concentraciones de oxígeno disuelto fueron mantenidas en muestras de suelo acuático, tasas de respiración por encima de $275-400 \mathrm{kgCO}_{2} / \mathrm{ha} / \mathrm{d}$.

Los suelos anegados tienen por lo general una menor tasa de respiración debido a que el agua ocupa los espacios porosos y el oxígeno atmosférico no puede penetrar rápidamente. Un exceso de agua por otro lado puede afectar el transporte y difusión de oxígeno hacia el interior de las partículas de suelo, creando condiciones anaeróbicas y reduciendo la respiración aeróbica (Orchard y Cook, 1983; Linn y Doran, 1984a, 1984b). La disminución de la humedad del suelo también afecta adversamente la actividad biológica y respiración de los suelos (Smith et al., 1993). La respiración de los suelos terrestres es comúnmente mayor al $60-80 \%$ de la capacidad de retención de humedad. A este contenido de humedad el suelo presenta un contenido de agua suficiente para la actividad microbial, ya que al- gunos poros no están saturados de agua y el aire puede ingresar para proveer oxígeno.

En la Tabla 1, se muestran los índices generales para diferentes niveles de respiración en el suelo según Woods End Research (1997). Un índice elevado de respiración del suelo es indicativo de una elevada actividad biológica y puede ser buen signo, indicativo de una rápida descomposición de residuos orgánicos hacia nutrientes disponibles para el crecimiento de las plantas. Sin embargo, la descomposición de la materia orgánica estable es perjudicial para diversos procesos físicos y químicos tales como agregación, intercambio catiónico y capacidad de retención de agua. Asimismo, inmediatamente a continuación de una operación de labranza, la evolución del $\mathrm{CO}_{2}$ puede acelerarse dramáticamente debido a la exposición de la materia orgánica a organismos y oxígeno (Volk, 1994).

$\mathrm{El} \mathrm{CO}_{2}$ de la rizósfera proviene en $1 / 3$ de la respiración de las raíces y $2 / 3$ de la respiración de los microorganismos. La producción de $\mathrm{CO}_{2}$ es afectado por el cultivo, labranza, fertilización, así como temperatura, humedad, aireación y pH del suelo. A nivel de la rizósfera se encuentra una concentración de $20,6 \%$ de oxígeno, menos de 0,20\% de $\mathrm{CO}_{2}$ y $79 \%$ de nitrógeno, el porcentaje de $\mathrm{CO}_{2}$ en la atmósfera es de sólo 0,03\%, es decir siete veces menos. En suelos compactos puede haber más de $10 \%$ de $\mathrm{CO}_{2}$ en la rizósfera. Sin embargo, contenidos mayores de $5 \%$ de $\mathrm{CO}_{2}$ y/o $<10 \%$ de oxígeno afectan el crecimiento de los cultivos agrícolas. El exceso de $\mathrm{CO}_{2}$ procura mayor solución de $\mathrm{CaCO}_{3}$, generando elevación en la concentración de bicarbonatos en la solución del suelo y esto a su vez provoca desórdenes nutricionales o fisiológicos en las plantas (Woods End Research, 1997).

Lacasta et al., (2006), estudiando el comportamiento durante tres años (2000-03) de la biomasa microbiana, la actividad respiratoria, la evolu- 
Tabla 1. Índices generales para clases de respiración del suelo, y estado del suelo, en condiciones óptimas de temperatura y humedad, primordialmente para uso agrícola (Woods End Research, 1997)

\begin{tabular}{|c|c|c|}
\hline $\begin{array}{l}\text { Respiración del suelo } \\
\mathrm{kg} \mathrm{C}\left(\mathrm{en} \mathrm{CO}_{2}\right) / \mathrm{ha} / \mathrm{d}\end{array}$ & Clase & Estado del suelo \\
\hline 0 & Sin actividad del suelo & $\begin{array}{l}\text { El suelo no presenta actividad biológica y es } \\
\text { virtualmente estéril }\end{array}$ \\
\hline$<10,64$ & $\begin{array}{l}\text { Actividad del suelo muy } \\
\text { baja }\end{array}$ & $\begin{array}{l}\text { El suelo ha perdido mucha materia orgánica disponible } \\
\text { y presenta poca actividad biológica. }\end{array}$ \\
\hline $10,64-17,92$ & $\begin{array}{l}\text { Actividad del suelo } \\
\text { moderadamente baja }\end{array}$ & $\begin{array}{l}\text { El suelo ha perdido parte de materia orgánica disponible } \\
\text { y la actividad biológica es baja. }\end{array}$ \\
\hline $17,92-35,84$ & $\begin{array}{l}\text { Actividad del suelo } \\
\text { mediana. }\end{array}$ & $\begin{array}{l}\text { El suelo se está aproximando, o alejando, de un estado } \\
\text { ideal de actividad biológica. }\end{array}$ \\
\hline $35,84-71,68$ & Actividad del suelo ideal & $\begin{array}{l}\text { El suelo se encuentra en un estado ideal de actividad } \\
\text { biológica y posee adecuada materia orgánica y activas } \\
\text { poblaciones de microorganismos. }\end{array}$ \\
\hline$>71,68$ & $\begin{array}{l}\text { Actividad del suelo } \\
\text { inusualmente alta. }\end{array}$ & $\begin{array}{l}\text { El suelo tiene un muy elevado nivel de actividad } \\
\text { microbiana y tiene elevados niveles de materia orgánica } \\
\text { disponible, posiblemente a través del agregado de } \\
\text { grandes cantidades de materia orgánica fresca o abonos. }\end{array}$ \\
\hline
\end{tabular}

ción de la materia orgánica y el nitrógeno mineral en dos suelos de texturas diferentes y sometidas a una rotación ecológica de barbecho-cebada-veza enterrada-trigo duro, llegó a las siguientes conclusiones: 1) Los suelos arcillosos tiene una mayor cantidad de biomasa microbiana, en cambio los franco-arenosos, al tener mayor aireación ponen a disposición de los cultivos mayor cantidad de nitrógeno mineral, por la mineralización de la materia orgánica. Esta mayor disponibilidad de nitrógeno permite producir una mayor cantidad de biomasa vegetal que se incorpora al sistema, permitiendo mantener los valores de materia orgánica y hacer sostenible el sistema. 2) Es con el uso del barbecho donde se dan las diferencias más grandes entre texturas arcillosas y francoarenosas en las disponibilidades de nitrógeno para el cultivo siguiente. En el suelo franco arenoso después de un barbecho y en los primeros $20 \mathrm{~cm}$ de suelo hay entre 75 y $120 \mathrm{~kg} / \mathrm{N} / \mathrm{ha}$ frente a los $30-75 \mathrm{~kg} / \mathrm{N} /$ ha de un suelo arcilloso, los márgenes dentro de un mismo suelo se deben a las condiciones pluviométricas del verano.

La investigación tuvo el objetivo de estudiar el efecto del abonamiento orgánico con estiércol de ganado vacuno, ovino, de cuy, fertilización química y sin abonamiento sobre la variación del nitrógeno, biomasa microbiana del suelo y la respiración del suelo en cultivos de papa y maíz

\section{MATERIALES Y MÉTODOS}

\section{Muestreo del suelo}

Considerando que la mayor cantidad de nutrientes y materia orgánica se ubican en los primeros $10 \mathrm{~cm}$ de profundidad, se colectaron muestras de aproximadamente $500 \mathrm{~g}$ de peso a 5 y $10 \mathrm{~cm}$ de profundidad, de cada una de las sub parcelas de papa y maíz, haciendo un total de 30 muestras. Sin embargo, para fines de ajustar a las posibili- 
dades logísticas y de análisis en laboratorio, las tres repeticiones fueron combinadas y tomadas como una sola, teniendo 10 muestras reales.

\section{Determinación de la respiración del suelo}

El método de incubación consistió en colocar a una muestra de $20 \mathrm{~g}$ de suelo en frascos herméticos de un litro que contienen $20 \mathrm{ml}$ de $\mathrm{KOH}$ a una concentración $2 \mathrm{M}$. Luego de un día de incubación el frasco de $\mathrm{KOH}$ es retirado y se añadió $\mathrm{BaCl}_{2}$ para detener la reacción con el $\mathrm{CO}_{2}$ ambiental. Luego, la solución fue titulada con $\mathrm{HCl}$ al 0,1 $\mathrm{N}$ para obtener las moléculas de $\mathrm{CO}_{2}$ emitidas por el suelo y capturadas en la solución (Alef y Nannipieri, 1995; García et al., 2003).

Un método adicional para esta determinación fue el de la utilización de la cámara dinámica cerrada (de PPSystems Inc. Inglaterra) que consta de dos componentes: 1 . La cámara de respiración del suelo, y 2. El medidor de $\mathrm{CO}_{2}$ basado en la absorción de la región infrarroja donde el $\mathrm{CO}_{2}$ tiene un máximo de absorción. Ambos equipos son utilizados para trabajo de campo y la cámara de respiración es insertada en el suelo y luego ésta realiza una circulación de aire a través del sistema cerrado y con ello se produce una acumulación. Este crecimiento de la concentración suele seguir una tendencia lineal con lo que luego es posible utilizarlo para determinar la pendiente y con ello la gradiente de concentración de $\mathrm{CO}_{2}$ respecto al tiempo.

\section{Cuantificación de la biomasa microbiana}

Se empleó el método de la fumigación - extracción. Primero se separaron muestras de $20 \mathrm{~g}$ de suelo a fumigar (F) y no - fumigar (NF). Estas últimas son introducidas en un desecador conteniendo al menos $10 \mathrm{ml}$ de cloroformo sin alcohol. El desecador es llevado al vacío por algunos minutos, para poder producir la ebullición del cloroformo, y así este pueda lograr una eficiente fumigación de los microbios del suelo expuesto. La fumigación promoverá la destrucción de las células microbianas que luego podrán ser extraídas mediante una solución de base débil de $\mathrm{K}_{2} \mathrm{SO}_{4}$ al $0.5 \mathrm{M}$. Ambas soluciones son analizadas para determinar los valores de C, N y P. Luego, la diferencia entre los valores de $\mathrm{F}$ y NF es utilizada para estimar la masa de microbios que estarían relacionados al respectivo elemento. La determinación de carbono se realizó empleando el método de Walkey - Black y el nitrógeno mediante el método Kjeldahl.

\section{Análisis estadístico}

Empleando análisis de variancia (ANVA) se determinó la significancia de los tratamientos; se realizó comparación múltiple Tukey para determinar el mejor tratamiento y mediante análisis de regresión y correlación se determinó la relación entre las variables en estudio.

\section{RESULTADOS Y DISCUSIÓN}

En la Tabla 2, se presentan los valores de nitrógeno en forma de nitrato, respiración basal del suelo $(\mathrm{RB})$ y biomasa microbiana del suelo (BM) en las localidades experimentales.

Los resultados del ANVA sobre contenido de nitrógeno (Tabla 3) indican diferencias significativas entre localidades en el cultivo de papa, mas no en maíz. La prueba de comparación múltiple (Tabla 4) muestra que en Chupaca existe significativamente menor disponibilidad que en las demás localidades. 
Tabla 2. Nitrógeno en forma de $\mathrm{NO}_{3}{ }^{-}$, respiración basal del suelo $(\mathrm{RB})$ y biomasa microbiana del suelo (BM) de cuatro localidades al final del cultivo de papa y maíz bajo cinco tipos de abonamiento (tratamiento)

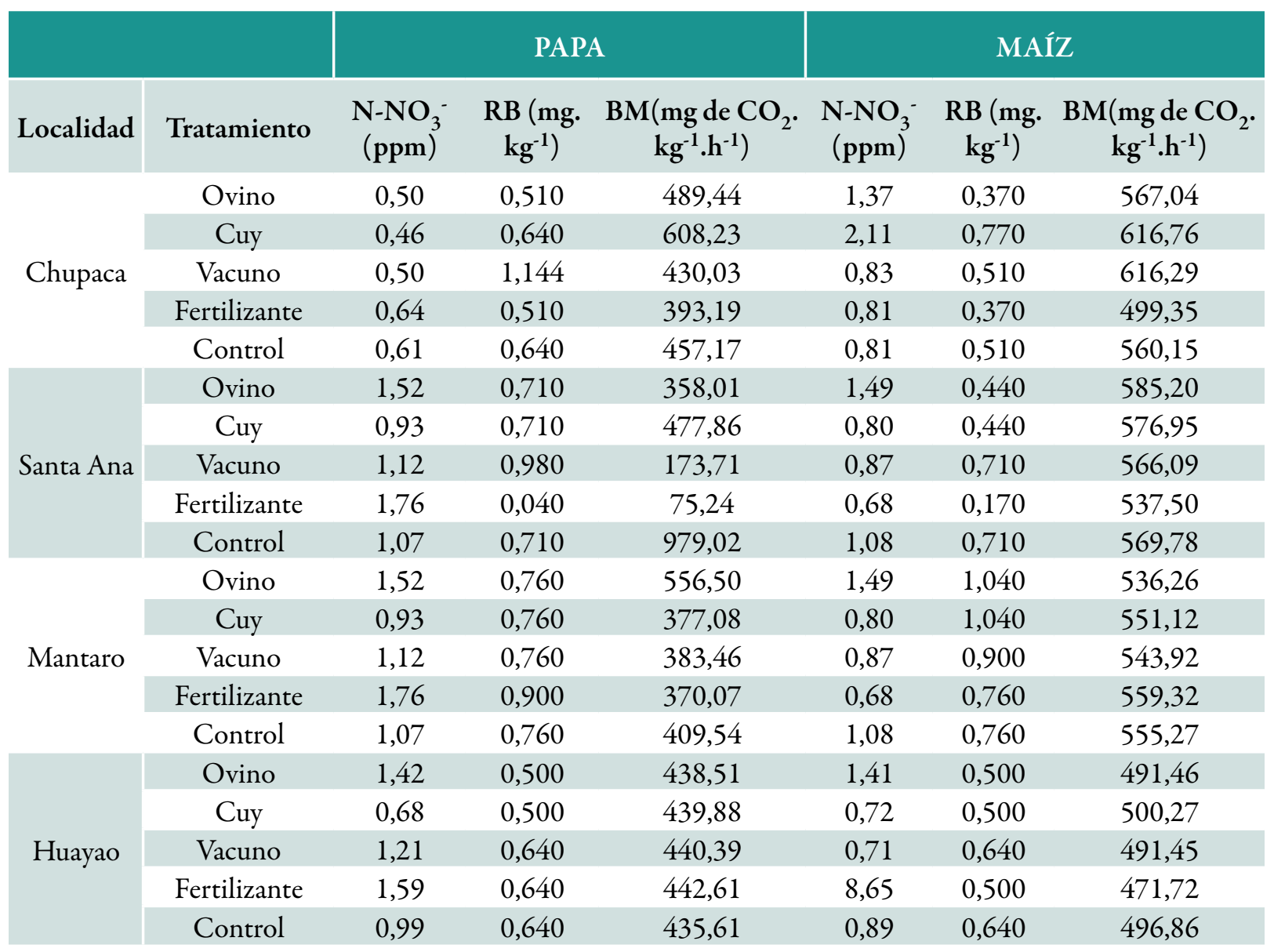

Tabla 3. Prueba de comparación múltiple para contenido promedio de $\mathrm{NO}_{3}{ }^{-}$, en el suelo de cuatro localidades al final del cultivo de papa y maíz bajo cinco tipos de abonamiento. Las letras indican diferencias significativas con una nivel $\alpha=0,05$

\begin{tabular}{c|ccccccc}
\multicolumn{2}{c}{ PAPA } & & & \multicolumn{3}{c}{ MAÍZ } \\
Localidad & Media & & & Localidad & Media & \\
\hline SANTA ANA & 1,280 & A & & HUAYAO & 2,476 & A \\
MANTARO & 1,280 & A & & CHUPACA & 1,186 & A \\
HUAYAO & 1,178 & A & & MANTARO & 0,984 & A \\
CHUPACA & 0,542 & & B & SANTA ANA & 0,984 & A \\
\hline
\end{tabular}


Tabla 4. Prueba de comparación múltiple para contenido promedio de $\mathrm{NO}_{3}{ }^{-}$en el suelo al final del cultivo de papa y maíz bajo cinco tipos de abonamiento. Las letras indican diferencias significativas con una nivel $\alpha=0,05$

\begin{tabular}{l|c|ccl|l|l|l} 
& PAPA & & & & \multicolumn{3}{c}{ MAÍZ } \\
Tratamiento & Media & & & & Tratamiento & Media & \\
Fertilizante & 1,438 & A & & & Fertilizante & 2,705 & A \\
Ovino & 1,240 & A & B & & Ovino & 1,440 & A \\
Vacuno & 0,988 & & B & C & Cuy & 1,108 & A \\
Control & 0,935 & & B & C & Control & 0,965 & A \\
Cuy & 0,750 & & & C & Vacuno & 0,820 & A \\
\hline
\end{tabular}

La tendencia del nitrógeno en todas las localidades es básicamente descendente. En Santa Ana y Mantaro se aprecia una ligera tendencia de ascenso hacia los 90 días, luego de haber descendido hacia los 60 días. Este comportamiento le proporciona la tendencia cúbica.
De modo general, la disponibilidad de nitrógeno en el suelo en el tiempo para el cultivo de papa sigue una moderada tendencia polinomial inversa de primer orden (Figura 1). Los altos valores iniciales se deben al aporte de nitrógeno en materia orgánica y en el fertilizante.

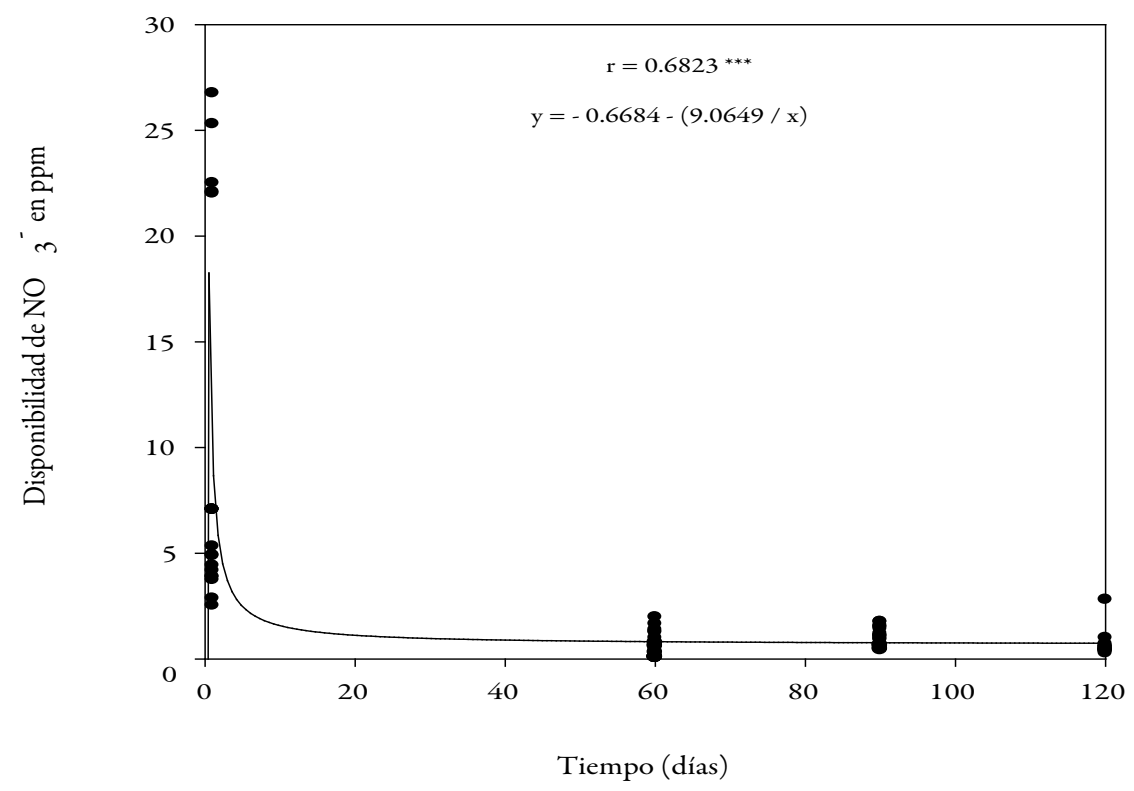

Figura 1. Tendencia de la disponibilidad de nitrógeno en forma de nitrato durante el periodo de cultivo de papa bajo cinco formas de abonamiento

Al igual que en cultivo de papa, de modo general, la disponibilidad de nitrógeno en el suelo en el tiempo para el cultivo de maíz sigue una moderada tendencia polinomial inversa de primer orden (Figura 2). Los altos valores iniciales también se deben al aporte de nitrógeno de la materia orgánica y el fertilizante. 


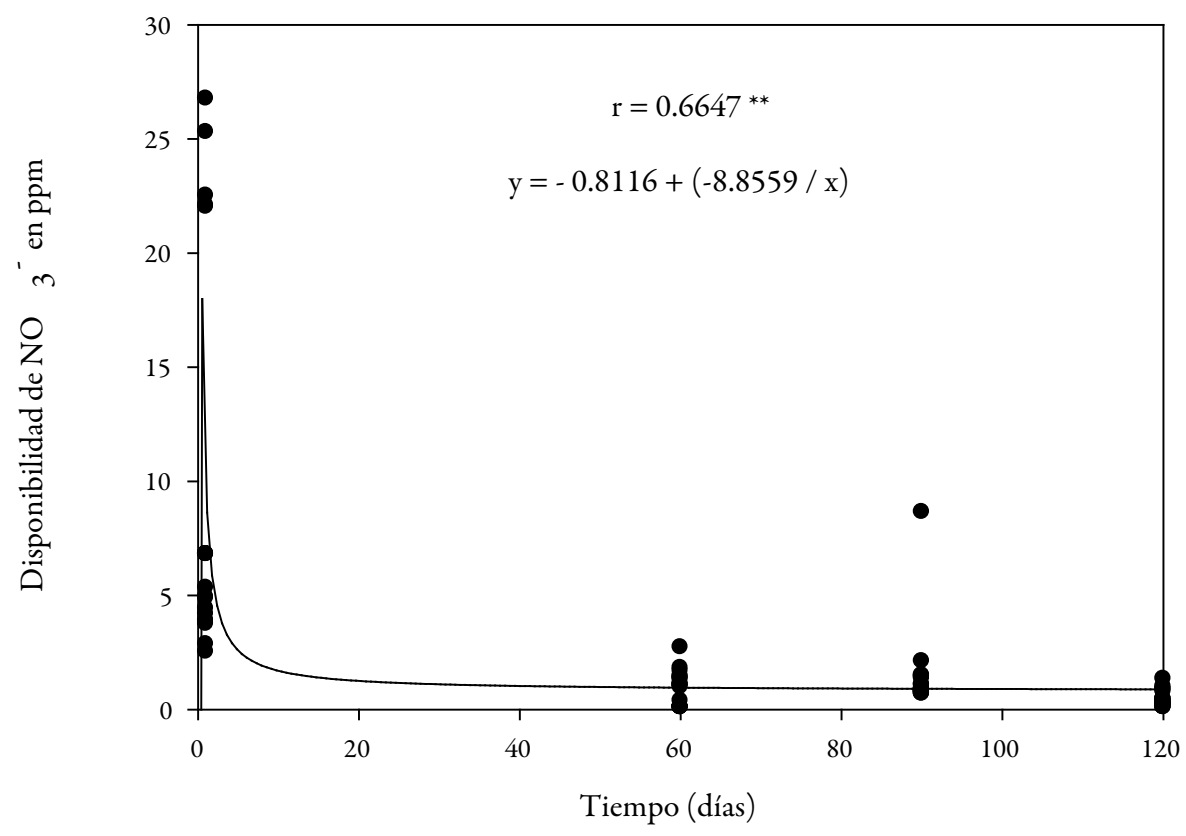

Figura 2. Tendencia de la disponibilidad de nitrógeno en forma de nitrato durante el periodo de cultivo de maíz bajo cinco formas de abonamiento

Los datos indican que no existe una relación definida entre la biomasa microbiana y la respiración. Tal como se puede apreciar en las figuras 3 y 4, la ausencia de relación definida se debe a los valores extremos y fuera de rango o

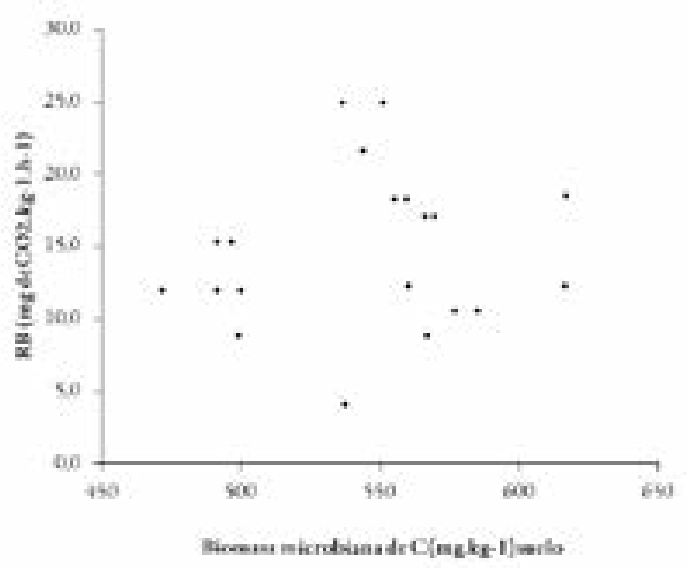

Figura 3. Biomasa microbiana vs Respiración basal en el cultivo de papa bajo cinco tipos de abonamiento quizás debido a errores en la determinación de las variables en estudio. El diagrama de dispersión entre la biomasa microbiana y respiración basal en el cultivo de papa, es similar al reportado por Islam y Weil (2000).

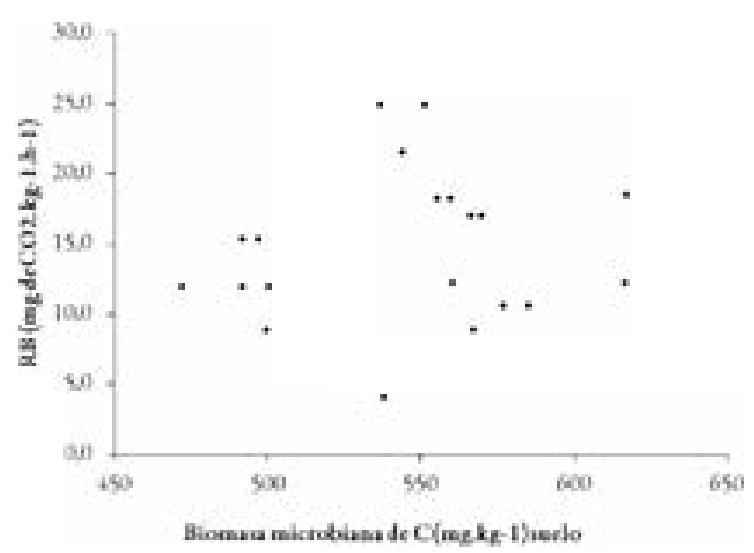

Figura 4. Biomasa microbiana vs Respiración basal en el cultivo de maíz bajo cinco tipos de abonamiento 
Las figuras 5 a 12, muestran las fluctuaciones de la respiración del suelo como resultado de la actividad de la biota presente en cada localidad y según tratamiento aplicado a los cultivos de papa y maíz.

Como se puede distinguir no existe un patrón de comportamiento, ya que cada localidad posee condiciones edáficas y ambientales propias que hacen posible la existencia de biotas diferentes y podríamos decir específicos, sin temor a equivocarnos ya que no hemos identificado

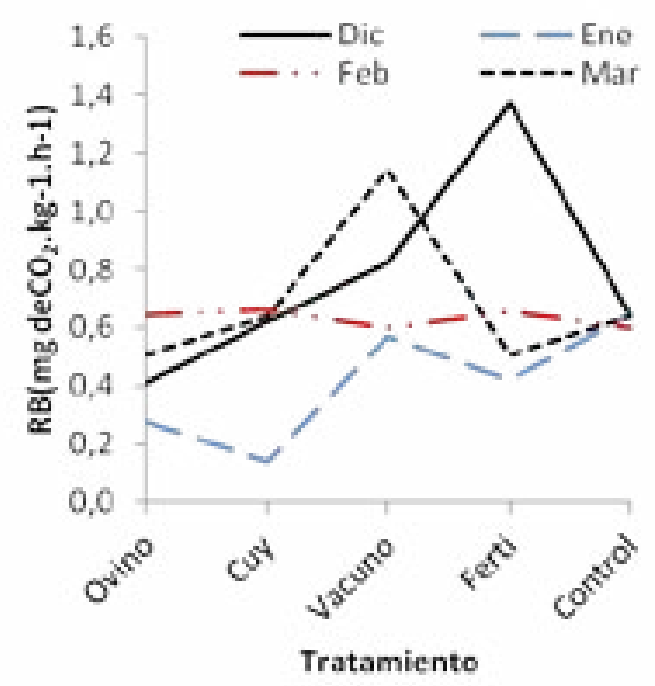

Figura 5. RB en Chupaca - Papa

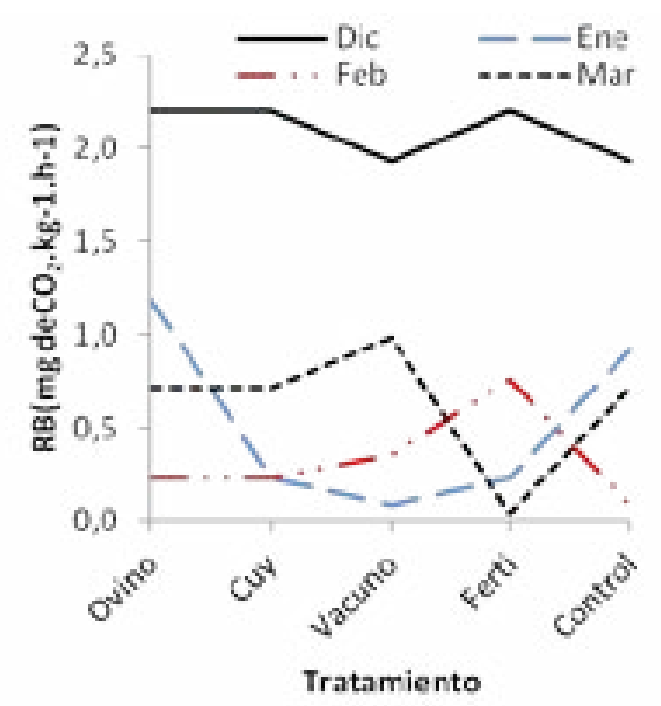

Figura 7. RB en Santa Ana - Papa especies y esta biota responde de manera diferente a los tratamientos y fluctuaciones ambientales, tal como lo plantea Moreira y Siqueira (2002).

Resultaría bastante extenso explicar el comportamiento en cada localidad. Esto sería tema de estudios posteriores para llegar a conclusiones y recomendaciones específicas y tal como plantea el USDA (1999) se debería determinar índices de respiración edáfica óptima, que determinen la cantidad de $\mathrm{C}$ perdido y la entrega de nutrientes (Parkinet et al., 1996)

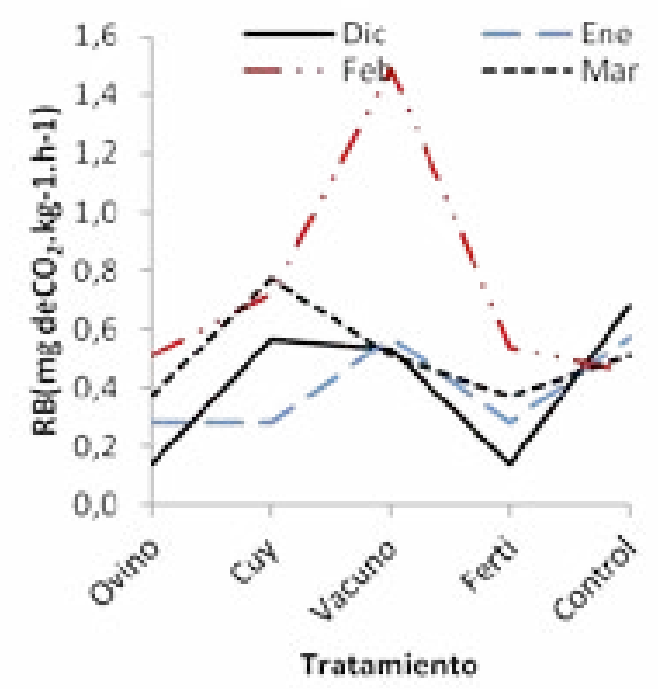

Figura 6. RB en Chupaca -Maíz

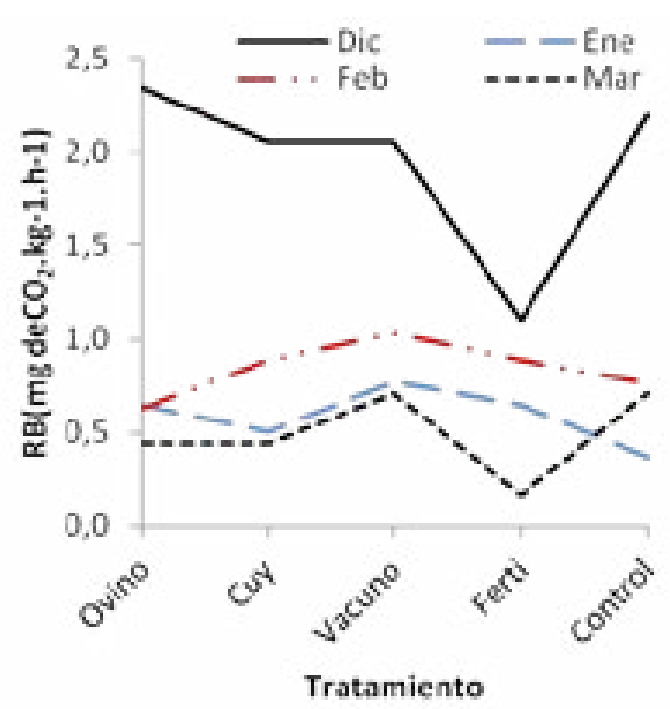

Figura 8. RB en Santa Ana - Maíz 


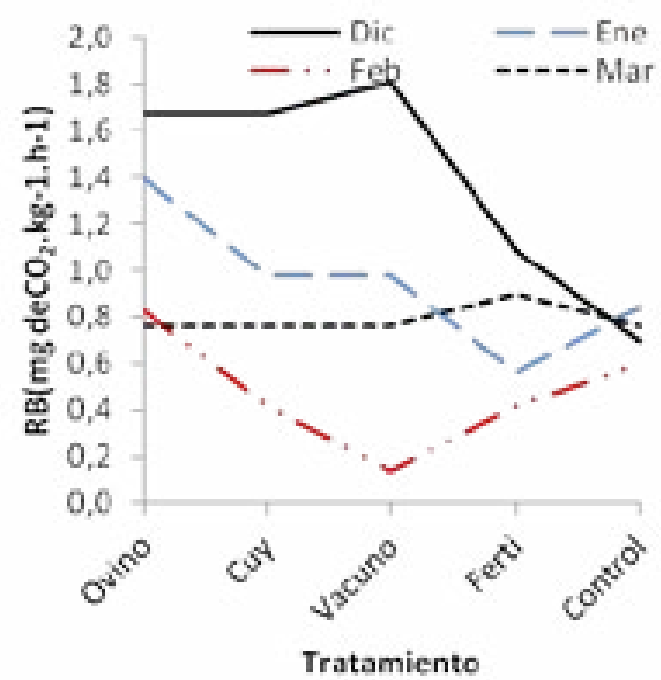

Figura 9. RB en El Mantaro - Papa

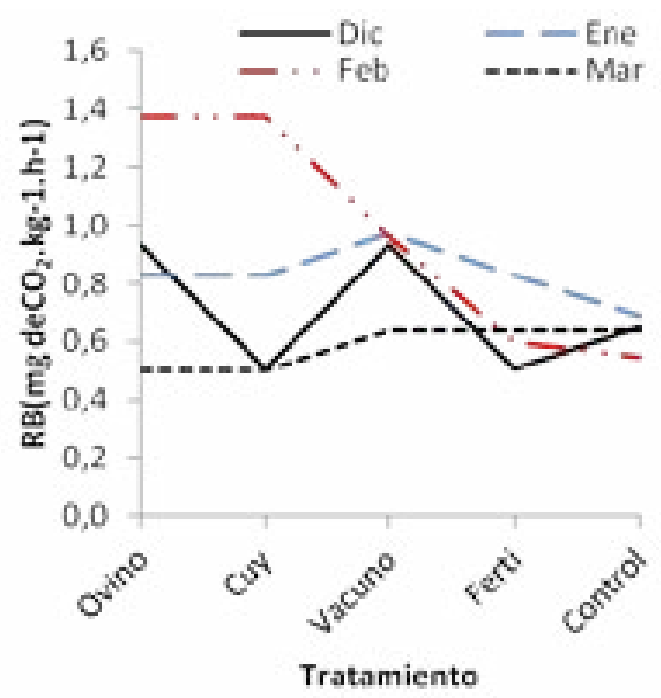

Figura 11. RB en Huayao -Papa

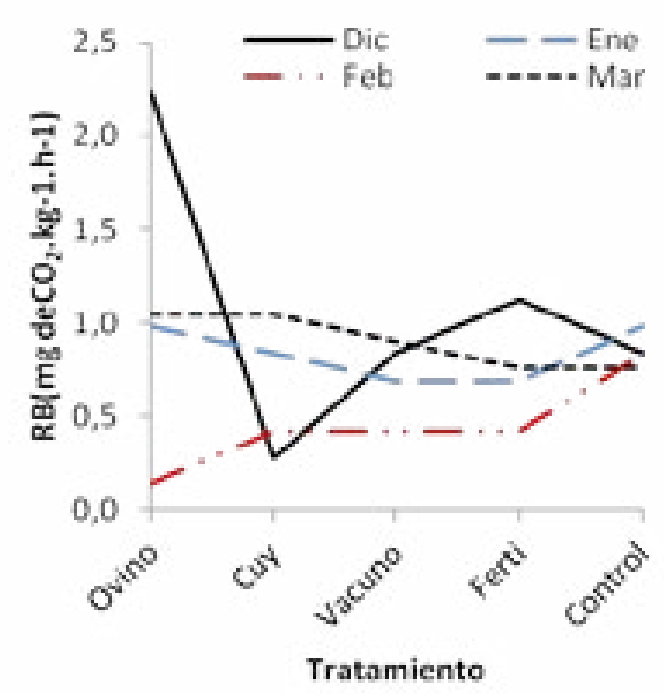

Figura 10. RB en El Mantaro - Maíz

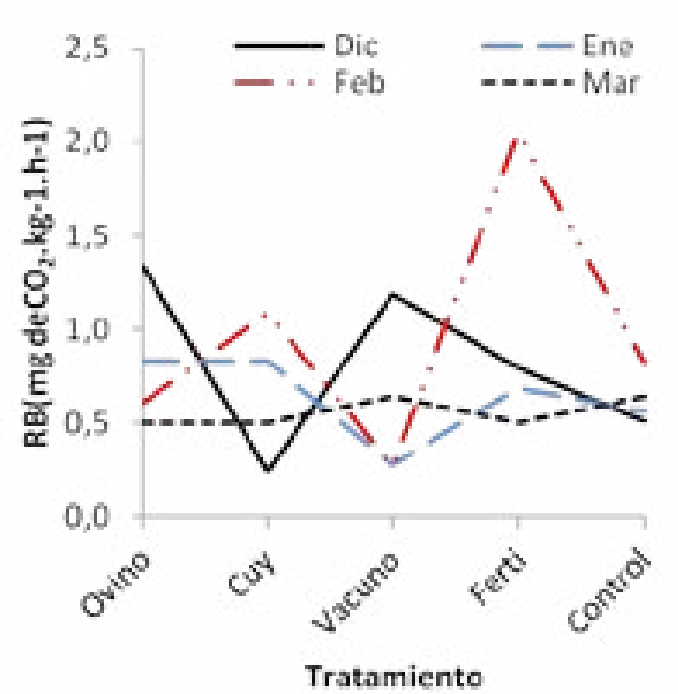

Figura 12. RB en Huayao - Maíz
El análisis de varianza para respiración del suelo en las cuatro localidades (Tabla 5) nos muestra diferencias significativas entre meses para las localidades de Santa Ana (en ambos cultivos) y para Mantaro (sólo en cultivo de papa). Estos resultados indicarían diferente nivel de actividad biológica en dichos suelos; mientras que en Chupaca y Huayao, la adición de materia orgánica no ha modificado de manera significativa la actividad biológica del suelo, en lo que se refiere a respiración.
Los resultados de análisis de correlación entre respiración en el suelo $(\mathrm{RB})$ y las variables temperatura $(T)$ a 5 y $10 \mathrm{~cm}$ de profundidad y humedad (Tabla 6), nos indica que existe una relación significativa pero inversa entre $\mathrm{RB}$ y $\mathrm{T}$ en la localidad de Chupaca en los dos cultivos y en Huayao para cultivo de maíz; mientras que en las demás localidades la relación es directa, es decir a mayor temperatura, mayor respiración en el suelo, esto resulta en mayor actividad biológica(Krebs, 2003; Thamdrup et al. 1998). 
Tabla 5. ANVA para respiración del suelo en cuatro localidades con cinco tipos de abonamiento durante cuatro meses de cultivo de papa y maíz

\begin{tabular}{|c|c|c|c|c|c|c|c|c|c|}
\hline \multirow{3}{*}{ F de V, } & \multirow{3}{*}{$\mathrm{g}, \mathrm{l}$} & \multicolumn{4}{|c|}{ Chupaca } & \multicolumn{4}{|c|}{ Santa Ana } \\
\hline & & \multicolumn{2}{|c|}{ Papa } & \multicolumn{2}{|c|}{ Maíz } & \multicolumn{2}{|c|}{ Papa } & \multicolumn{2}{|c|}{ Maíz } \\
\hline & & $\mathrm{CM}$ & $\mathrm{P}$ & $\overline{\mathrm{CM}}$ & $\mathrm{P}$ & $\mathrm{CM}$ & $\mathrm{P}$ & $\mathrm{CM}$ & $\mathrm{P}$ \\
\hline Tratamiento & 4 & 0,077 & 0,303 & 0,143 & 0,081 & 0,049 & 0,8288 & 0,105 & 0,3055 \\
\hline Mes & 3 & 0,120 & 0,150 & 0,127 & 0,118 & 3,249 & $<0,0001$ & 2,252 & $<0,0001$ \\
\hline \multirow{3}{*}{ F de V, } & \multirow{3}{*}{$\mathrm{g}, 1$} & \multicolumn{4}{|c|}{ Mantaro } & \multicolumn{4}{|c|}{ Huayao } \\
\hline & & \multicolumn{2}{|c|}{ Papa } & \multicolumn{2}{|c|}{ Maíz } & \multicolumn{2}{|c|}{ Papa } & \multicolumn{2}{|c|}{ Maíz } \\
\hline & & $\mathrm{CM}$ & $\mathrm{P}$ & $\mathrm{CM}$ & $P$ & $\mathrm{CM}$ & $\mathrm{P}$ & $\mathrm{CM}$ & $\mathrm{P}$ \\
\hline Tratamiento & 4 & 0,129 & 0,259 & 0,125 & 0,573 & 0,065 & 0,332 & 0,116 & 0,689 \\
\hline Mes & 3 & 0,711 & 0,003 & 0,341 & 0,158 & 0,137 & 0,094 & 0,169 & 0,501 \\
\hline
\end{tabular}

Este comportamiento contrario indicaría que Chupaca y Huayao poseen una biota menos exigente en temperaturas altas, mientras que en Santa Ana y Mantaro, la biota del suelo es más activa a mayor temperatura, ya que los suelos son de colores claros y retienen menos la radiación solar. La humedad, la intensidad de la radiación solar por unidad de superficie, la evaporación, convección, la exposición, la sombra y el aislamiento son importantes factores que regulan la temperatura del suelo (Hagan, R y McCalla 1952, citado por Ochse, et al, 1991). El cultivo de papa logra una mayor cobertura que el maíz, razón por la cual los suelos con cultivo de papa al estar menos expuestos directamente por la radiación solar se calientan menos que los suelos con cultivo de maíz, aunque en las primeras horas de la mañana y últimas de la tarde las plantas proporcionan mayor sombra que la del cultivo de papa, cuando los cultivos logran su máximo crecimiento.

Relación alta y significativa entre respiración en el suelo y humedad sólo se ha determinado en la localidad de Chupaca. Esta falta de relación en la mayoría de casos se debe al comportamiento no normal de la humedad como factor meteorológico y en este caso como factor edáfico.
Según Bowman y Delfino (1980), rangos usuales de respiración se encuentran entre $10-100$ $\mathrm{kgCO}_{2} / \mathrm{ha} / \mathrm{h}$,pero en la mayoría de suelos se encuentran en el rango $25-40 \mathrm{kgCO}_{2} / \mathrm{ha} / \mathrm{d}$, que corresponden a 1,04 y $1,67 \mathrm{kgCO}_{2} / \mathrm{ha} / \mathrm{h}$ calificados como medios por Woods End Research (1997), concuerdan con los determinados en el presente estudio y sólo en diciembre alcanzan el nivel ideal (Figuras 13 y 14). Los valores de respiración del suelo muestran variabilidad por efecto de la adición de materia orgánica (Volk, 1994) y del fertilizante químico, más no existen grandes variaciones en los tratamientos control en todas las localidades donde se presenta una actividad del suelo moderadamente baja, excepto en el cultivo de maíz en Santa Ana, resultado que debería ser estudiado en el futuro. Para Chupaca y Huayao la adición de materia orgánica ha dado como resultado desde muy baja hacia mediana actividad en ambos cultivos, resultando un tanto mayor en el mes de diciembre y menor en marzo. Esto se explica porque en el mes de diciembre se incrementa la temperatura y en los siguientes meses la precipitación la regula y en marzo definitivamente desciende la temperatura y por ende la actividad microbiana (Orchard y Cook, 1983; Linn y Doran, 1984a, 1984b; Smith et al. 1993) 
En Santa Ana durante el mes de diciembre se producen rangos de respiración ideales en el suelo con ambos cultivos y muy baja actividad durante marzo por diferencias pluviométricas (Lacasta et al., 2006). Para el caso de Mantaro, en el cultivo de papa se presenta una actividad microbiana ideal durante el mes de diciembre bajo los tratamientos con estiércol y en maíz bajo estiércol de ovino. El resto de meses la biota del suelo muestra, de muy baja a media actividad.

Tabla 6. Resumen de los coeficientes de correlación significativos y algunos mayores de $\alpha=0,1$ entre la variable respiración (RB) en el suelo, versus los variables temperatura a $5 \mathrm{~cm}(\mathrm{~T} 5)$, temperatura a $10 \mathrm{~cm}(\mathrm{~T} 10)$ de profundidad y humedad del suelo $(\mathrm{H})$ en la diferentes localidades, durante todo el periodo vegetativo (Todo) y por meses

\begin{tabular}{|c|c|c|c|c|c|c|c|}
\hline Chupaca & Enero & Santa Ana & Todo & Mantaro & Todo & Huayao & Febrero \\
\hline Papa & $\mathrm{RB}$ & Papa & $\mathrm{RB}$ & Papa & $\mathrm{RB}$ & Papa & $\mathrm{RB}$ \\
\hline \multirow[t]{2}{*}{$\mathrm{T} 5$} & $-0,8865$ & $\mathrm{~T} 5$ & 0,8493 & $\mathrm{~T} 5$ & 0,5437 & $\mathrm{~T} 5$ & 0,7211 \\
\hline & 0,0451 & & $<, 0001$ & & 0,0132 & & 0,1693 \\
\hline \multirow[t]{2}{*}{ T10 } & $-0,9601$ & $\mathrm{~T} 10$ & 0,8208 & $\mathrm{~T} 10$ & 0,4217 & $\mathrm{~T} 10$ & 0,9176 \\
\hline & 0,0095 & & $<, 0001$ & & 0,0640 & & 0,0280 \\
\hline \multirow[t]{2}{*}{$\mathrm{H}$} & 0,9387 & $\mathrm{H}$ & 0,0742 & $\mathrm{H}$ & $-0,4000$ & $\mathrm{H}$ & 0,6414 \\
\hline & 0,0180 & & 0,7559 & & 0,0806 & & 0,2434 \\
\hline Chupaca & Todo & Santa Ana & Todo & & & Huayao & Diciembre \\
\hline Maíz & $\mathrm{RB}$ & Maíz & $\mathrm{RB}$ & & & Maíz & $\mathrm{RB}$ \\
\hline \multirow[t]{2}{*}{ T5 } & $-0,5141$ & $\mathrm{~T} 5$ & 0,9058 & & & $\mathrm{~T} 5$ & $-0,1676$ \\
\hline & 0,0204 & & $<, 0001$ & & & & 0,7876 \\
\hline \multirow[t]{2}{*}{$\mathrm{T} 10$} & $-0,6408$ & $\mathrm{~T} 10$ & 0,8731 & & & $\mathrm{~T} 10$ & $-0,9558$ \\
\hline & 0,0023 & & $<, 0001$ & & & & 0,0111 \\
\hline \multirow[t]{2}{*}{$\mathrm{H}$} & 0,3404 & $\mathrm{H}$ & $-0,1350$ & & & $\mathrm{H}$ & 0,8272 \\
\hline & 0,1419 & & 0,5705 & & & & 0,0840 \\
\hline Chupaca & Febrero & & & & & & \\
\hline Maíz & $\mathrm{RB}$ & & & & & & \\
\hline \multirow[t]{2}{*}{ T5 } & $-0,5932$ & & & & & & \\
\hline & 0,2917 & & & & & & \\
\hline \multirow[t]{2}{*}{ T10 } & $-0,8510$ & & & & & & \\
\hline & 0,0675 & & & & & & \\
\hline \multirow[t]{2}{*}{$\mathrm{H}$} & 0,3572 & & & & & & \\
\hline & 0,5551 & & & & & & \\
\hline
\end{tabular}

Nota: Los valores bajo los coeficientes de correlación $\mathrm{r}$, indican el nivel de significación 

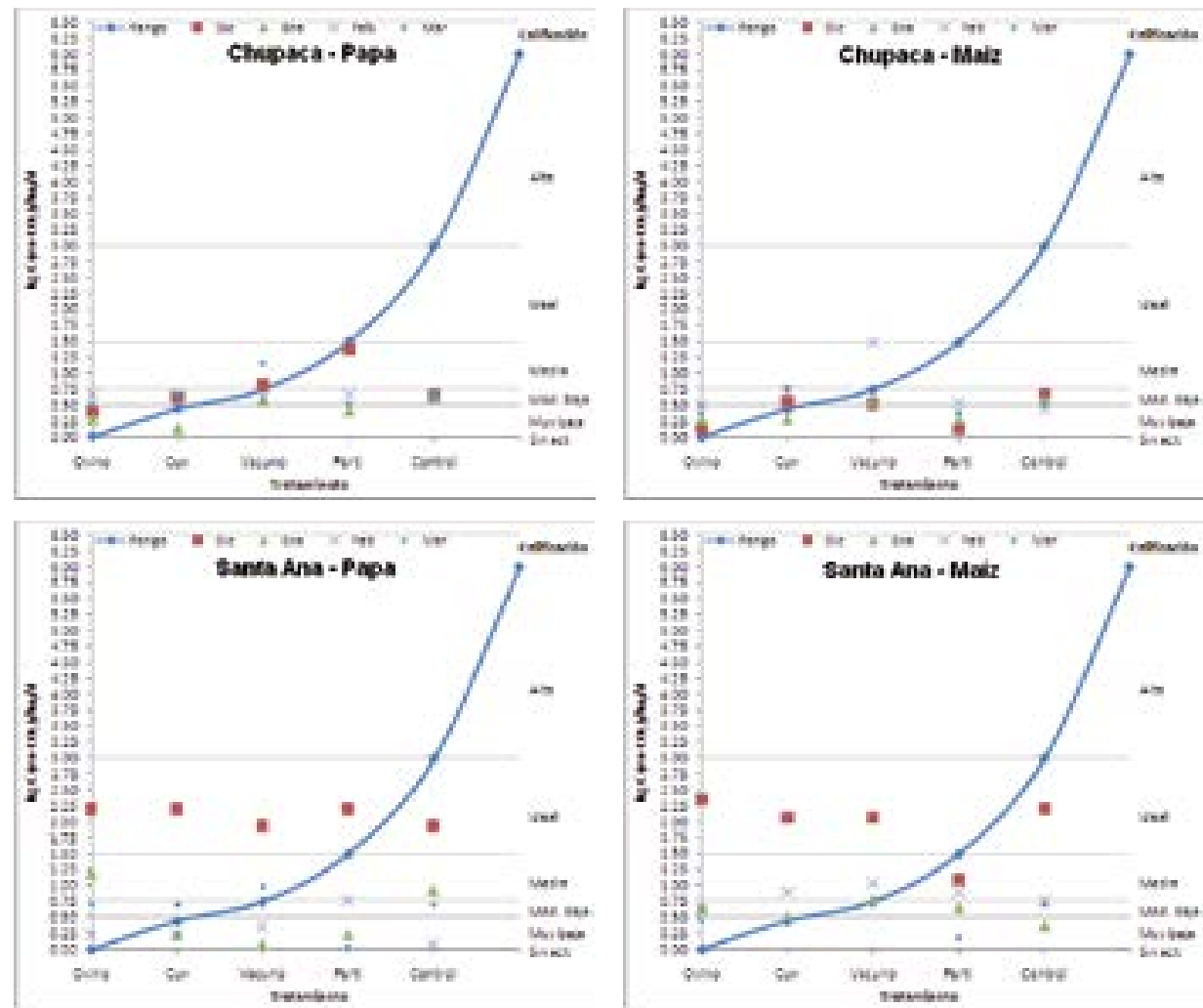

Figura 13. Calificación de la respiración del suelo en los cultivos de papa y maíz bajo cinco tratamientos de fertilidad en localidades de Chupaca y Santa Ana
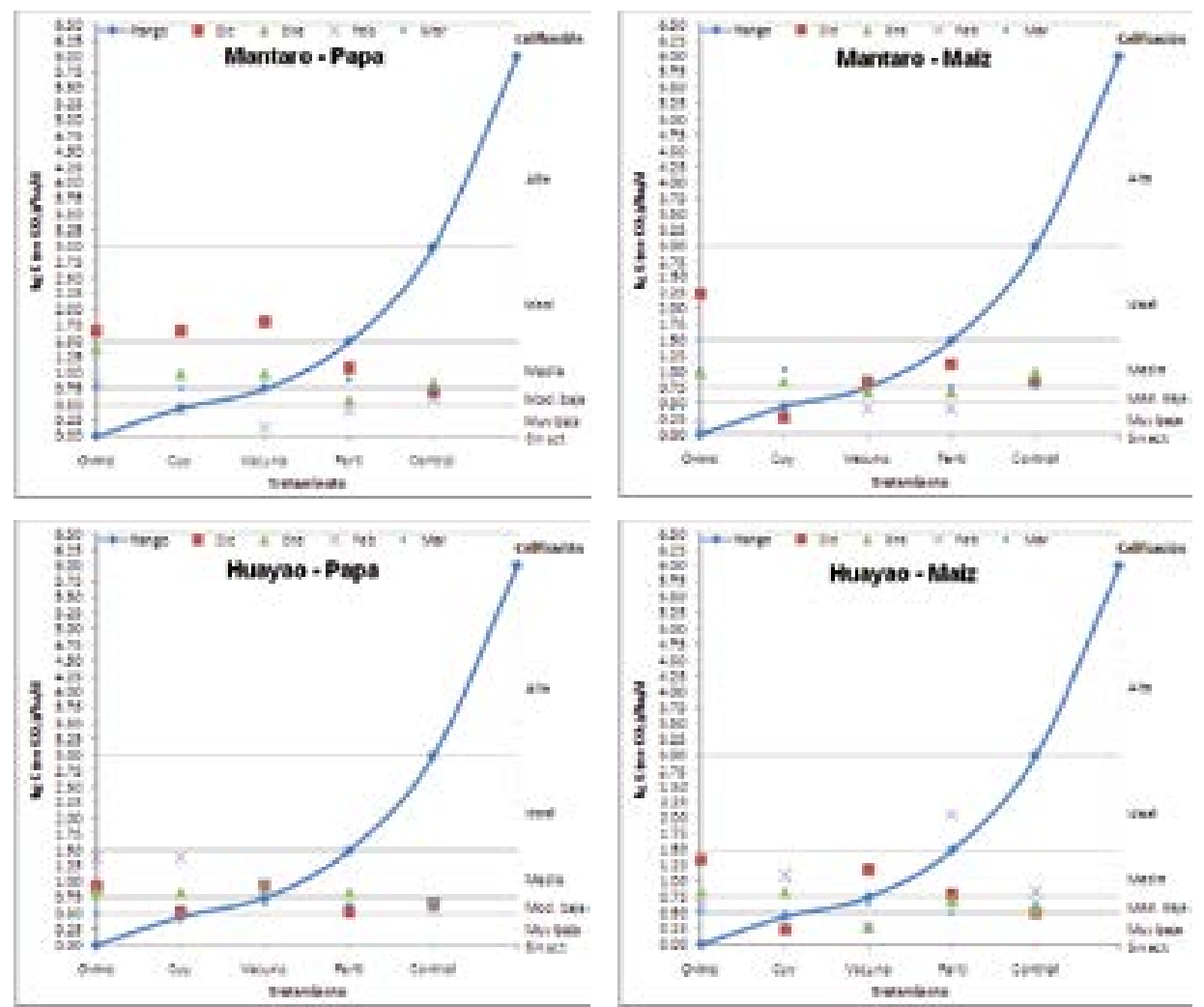

Figura 14. Calificación de la respiración del suelo en los cultivos de papa y maíz bajo cinco tratamientos de fertilidad en localidades de Mantaro y Huayao 


\section{CONCLUSIONES}

1. La variación del nitrógeno es de tendencia polinomial mostrando una continua disminución hacia los 60 días y un ligero ascenso hacia los 90 días.

2. La actividad microbiológica es de muy baja a media en la mayoría de suelos y tratamientos, presentándose la mayor tasa de respiración durante el mes de diciembre, tiempo en el cual existe mayor temperatura en el suelo con respecto a los meses de febrero y marzo. Es decir a mayor temperatura mayor respiración basal.

3. No existe una relación definida entre la biomasa microbiana y la respiración del suelo.

4. Resulta bastante extenso explicar el comportamiento de la RB en cada localidad. Esto sería tema de estudios posteriores para llegar a conclusiones y recomendaciones específicas.

\section{BIBLIOGRAFÍA}

1. Alef,K.; Nannipieri, P. 1995. Methods in applied soil microbiology and biochemistry. Academic Press, London.

2. Bowman, G. T., y J. J. Delfino. 1980. Sediment oxygen demand techniques: A review and comparison of laboratory and in situ systems. Water Res. 14: 491-499.

3. García, C.; Gil-Sotres, F.; Hernández, T.; Trasar-Cepeda, C. 2003. Técnicas de análisis de parámetros bioquímicos en suelos: medida de actividades enzimáticas y biomasa microbiana. Mundi-Prensa, Madrid. 371 p.

4. Islam, K.R. and R.R.Weil. 2000. Soil quality indicator properties-in mid-Atlantic soils as influenced by conservation management. Soil and Water Conservation Society.Journal of Soil and WaterConservation 55(1):69-78

5. Jenkinson, D.S. 1988. Soil organic matter and its dynamics.In: Wild, A. (Ed.). Russel's soil conditions and plant growth. 11 th ed. Longman. New York, USA. p. 564-607.

6. Krebs Reginatto, Luciano. 2003. "Respiración del suelo como herramienta para evaluar calidad de fondos en acuicultura. I. Desarrollo de un protocolo estándar para medir dióxido de carbono". Tesis de grado Magister en ciencias. Escuela Superior Politécnica del Litoral. Facultad de Ingeniería Marítima y Ciencias del Mar. Guayaquil - Ecuador. 67p

7. Lacasta, C.; M. Benítez; N. Maire; y R. Meco. 2006. Efecto de la textura del suelo sobre diferentes parámetros bioquímicos. VII Congreso SEAE: Agricultura y Alimentación Ecológica. Toledo, España.

8. Linn, D. M., y J. W. Doran. 1984a. Aerobic and anaerobic microbial populations in notill and plowed soil.Soil Science Society of America Journal 48: 794-799.

9. Linn, D. M., y J. W. Doran. 1984b. Effect of water-filled pore space on carbon dioxide and nitrous oxide production in tilled and nontilled soils. Soil Science Society of America Journal 48: 1257-1272.

10. Moreira, M. M. S., y Siqueira, J. O, 2002. Microbiología e bioquímica do solo. Ed. UFLA. Brasil.

11. Ochse, J.; M.J. Sole; M.J. Dijkman; C. Wehlburg. 1991. Cultivo y mejoramiento de plantas tropicales y subtropicales. Vol I. Edit. Noriega_ Limusa. México, 828 pp.

12. Orchard, V. A., y F. J. Cook. 1983. Relationship between soil respiration and soil moisture. Soil Biology \& Biochemistry 15: 447-454.

13. Parkin, T.B., J.W. Doran, and E. FrancoVizcaino. 1996. Field and laboratory tests of soil respiration. P. 231-246. In: J.W. Doran and A.J. Jones (eds.) Methods for assessing soil quality. Soil Sci. Soc. Am. Spec. Publ. 49. SSSA, Madison, WI.

14. Smith, J. L., R. I. Papendick, D. F. Bezdicek, y J. M. Lynch. 1993. Soil organic matter dynamics and crop residue management. In Soil Microbial Ecology, F. B. Metting, Jr., ed., pp $65-94$. 
Marcel Dekker, New York.

15. Thamdrub, B. J. W. Hansen, y B. B. Jorgensen. 1998. Temperature dependence of aerobic respiration in a coastal sediment. Microbiology Ecology. 25:189-200.

16. USDA 1999. Departamento de Agricultura de los Estados Unidos de América. Guía para la evaluación de la calidad y salud del suelo. Traducción al español por Juan Carlos Salazar y Alberto Luters. Instituto de Suelos. Argentina. Julio 2000. p: 31-54

17. Volk, T. 1994. The soil's breath. Natural history November/94.

18. Woods End Research. 1997. Guide to solvita testing and managing your soil. Woods EndResearchLaboratory, Inc., Mt. Vernon, ME

\section{Correspondencia:}

\section{Luis Suarez}

email:luchinsuarez@yahoo.com

\section{AGRADECIMIENTO}

Esta investigación fue financiada por el Fondo de Innovación Científica y Tecnológica (FINCYT) del Gobierno de Perú en merced al contrato 028-FINCYT-PIBAP-2007 del proyecto "Estudio de la diversidad y biomasa microbiana del suelo para mejorar la calidad de la materia orgánica de tierras agrícolas". Se agradece a la Estación Experimental "Santa Ana” del Instituto Nacional de Innovación Agraria (INIA) por el uso de sus áreas experimentales. 